\title{
HBB4ALL: Deployment of HbbTV Services for All
}

\author{
Pilar Orero, Carlos Alberto Martín,
}

Mikel Zorrilla

\begin{abstract}
Hybrid Broadcast Television for All is a European Commission co-financed project, inside the Competitiveness and Innovation Framework Programme (CIP). The project builds on HbbTV, the European standard for broadcast and broadband multimedia converged services, and looks at how HbbTV technology may be used to enhance access services (such as subtitling, audio description or sign language) on both the production and service sides. HbbTV 1.5 devices are widely available in the market while HbbTV version 2.0 specification has been recently released. TV content can be enhanced by HbbTV applications with additional synchronised services in a personalised manner. For access services this opens an entirely new opportunity for users who may choose an access service delivered via their IP connection which seamlessly integrates with the regular broadcast programme. The presentation will describe the improvements taken on board by HBB4ALL to existing access services and ways of addressing the key technical, organisational and legal obstacles to the sustainable take-up of these services throughout Europe. HBB4ALL focuses on real pilot deployment as a first step to ensure a successful exploitation of these services in a near future. We will offer new insights, from the fields of human machine interaction and social innovation, which arise from the new interactive multimodal and multilanguage services which may be offered. This article will first describe the structure chosen for the project, with four pilots developed in parallel: subtitling, audio description, sign language and user interaction. Then it will describe the methodology and research approaches used for testing the new accessibility services.
\end{abstract}

Index Terms-IPTV \& Internet TV, objective evaluation techniques, access services, accessibility, HbbTV, Connected TV

\section{INTRODUCTION}

$\mathrm{T}$ HE deployment of HbbTV in Europe represents both a challenge and an opportunity for access services. Existing services such as: subtitling, audio description, audio subtitling and sign language can be produced, broadcast and received in many formats and on many different device types. HbbTV technology may be used to enhance both linear and Video On Demand TV by synchronising broadcast and broadband content. For access services this opens up new opportunities to provide personalised access services delivered via IP connection which seamlessly integrate with the regular broadcast programmes. This paper will first describe the structure chosen for the project, with four pilots developed in parallel: subtitling, audio description, sign language and user interaction. Then it will describe the methodology and research approaches testing the new accessibility services. It will finally describe the improvements to existing access services and ways of addressing the key technical, organisational and legal obstacles to the sustainable take-up of these services throughout Europe. The HBB4ALL project and the HbbTV standard prove how broadcast and broadband technologies can be efficiently and effectively merged to provide innovative and enriched multimedia services.

\section{Project Structure}

The project will test access services in various pilot implementations (from the definition to the operational phase) and gather implicit and explicit user feedback to assess the acceptance and the achievable quality of service in the various delivery scenarios (broadcasting, hybrid and full IP).

Four interlinked pilots (Fig.1) with a common framework defined by a "joint cross-pilot coordination" (Fig. 2) are implemented in HBB4ALL:

- Pilot A: Multi-platform subtitle workflow chain

- Pilot B: Alternative audio production and distribution

- Pilot C: Automatic UI adaptation - accessible Smart TV applications

- Pilot D: Sign-language translation service

The four pilots are worked across in five defined tasks common to all pilots. The first deals with the overall coordination of all tasks and is related to managerial issues. The second task looks at the many technical components implemented in the project and how to share them to optimize performance. The methodology used was first to create an overview of existing or planned technologies. In the future, we will use this information as a basis for further discussions with the aim to identify potential common technical solutions and other technical development synergies. For example in subtitling, the workflow can be divided into three parts, each 
of which needs dedicated technical implementations: Authoring, Standards and Technologies, and Contribution.

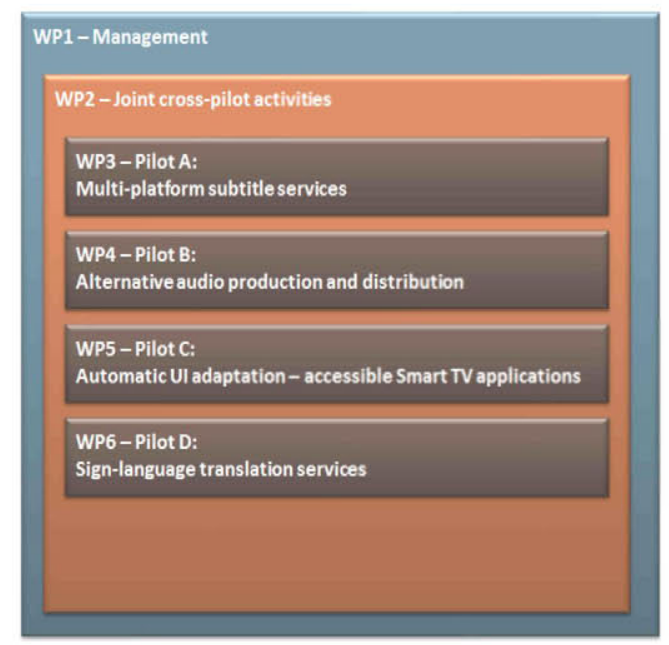

Fig 1. Project structure

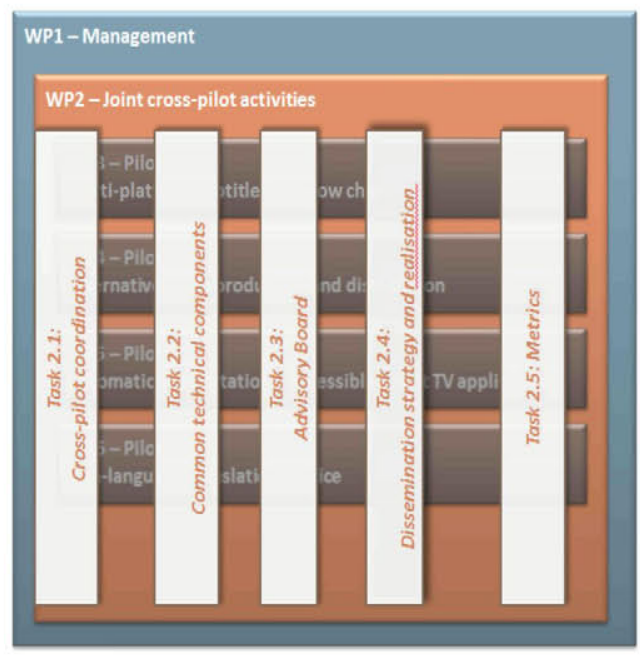

Fig. 2 Project structure with cross-activities

\section{A. Authoring}

Authoring covers the creation of the subtitles. This is done either: by a person with the help of a preparation tool, or automatically by using speech recognition technologies. HBB4ALL will build on existing systems and will advance some of them with prototypical features to support a multiplatform workflow. A preparation tool that has the potential to be extended with these features is WinCaps Qu4ntum by the HBB4ALL partner Screen Subtitling Systems. As additional reference for generic, vendor independent strategies prototypical reference software modules are provided. Essential for the adoption of a multiplatform workflow is the information model used for subtitle information; for prepared programs this involves the file based formats used and for live programs the applied network protocols. Apart from vendor specific file based subtitle formats EBU STL acts as an interoperable solution. For live programs several proprietary protocols are used. Most of them are derived from the NewFor Protocol. Both EBUSTL and NewFor have the disadvantage that they are not easy to extend and that they are restricted to subtitle information for teletext subtitles. In HBB4ALL the XML format EBU-TT will be used in prototypical implementations. EBU-TT can carry subtitle information that is adequate for more than one platform and allows the addition of metadata which can consequently be used in the HBB4ALL multiplatform workflow.

HBB4ALL will provide a guideline document on how to use EBU-TT in the authoring context and define additional metadata that can be used in the different pilots. Extensions of WinCAPS Qu4ntum could be implemented to support EBUTT as an export format. In addition an open source software module will be provided that transcodes the currently used EBU STL format into EBU-TT. Additional EBU-TT transformation modules are implemented to add pilot specific metadata to an EBU-TT document.

Depending of the requirement analysis a prototypical preview feature will be provided to verify the presentation of created subtitles on different platforms. This could be done in WinCAPS and/or an external EBU-TT preview tool that simulates various presentations with web technologies (HTML, Javascript and CSS). For Live Subtitles a replacement of the NewFor protocol could be defined. This could be a part of the preparation tool and/or could be handled by an external module that expects NewFor as input and provides a stream of EBU-TT documents as output.

\section{B. Standards and Technologies}

The standards and specifications involved in subtitling authoring and storing are:

- Tech 3350, EBU-TT Part 1 Subtitling format definition, Version 1.0

- TECH 3360 MAPPING EBU STL (TECH 3264) TO EBU-TT SUBTITLE FILES, Draft for Comment, Version 0.9

- Tech 3264-1991 EBU subtitling data exchange format

- Timed Text Markup Language 1 (TTML1) (Second Edition), W3C Recommendation 24 September 2013

- Newfor Protocol for Teletext Subtitles (various proprietary specifications)

\section{Contribution}

The contribution part of the subtitle workflow covers the exchange and the transmission of subtitling information before it gets distributed to end devices. Some of the formats from the authoring context (EBU STL and Newfor) are also used here and therefore all implementations for the authoring part can also be used in the contribution part.

Current technologies that are used for the in-band transmission of subtitle information (VBI in SDTV and 
VANC in HDTV) have to be considered. It is unlikely that this operational part of the workflow will be changed in HBB4ALL although the transport of EBU-TT documents in the VANC could be tested dependent of the support of vendor tools. Contributed subtitles information for the transmission of teletext and DVB subtitles have to guarantee that these services are fully supported.

To distribute subtitles for broadcast content to an HbbTV application, a system must contribute subtitle information to a MPEG2-TS multiplexer. A system that is currently used by RBB for this purpose is the iScheduler of the company MITxperts. The contribution of live subtitles information for broadband distribution may require a mix of existing technologies that cannot be fully be specified at this point in time.

The third task in the cross-coordination approach is to have an Advisory Board with six experts at EU level representing stakeholders in the project: two user associations: European Disability Forum (EDF), and Age Platform Europe, two technical standardisation partners the European Broadcasting Union (EBU) and the International Telecommunication Union, manufacturers are represented by Digital Europe and the EU accessibility network eAccess + .

\section{TEST AND PILOTS}

The project will work at two levels. On the one hand small tests with end users will take place during the first year of the project in lab conditions across Europe. On the other hand, large scale pilots will be deployed to test the service performance in real conditions. For this purpose, relevant broadcasters are involved in the HBB4ALL consortium and will offer the service for a period of time while feedback is gathered from end users.

Tests have been designed using a bottom-up approach, which is from user feedback to broadcasters on existing services, or by broadcasters' anticipation of HbbTV services. In order to be comprehensive and representative of the European broadcast languages, the three European translation traditions will be covered in all tests: voice over, dubbing and subtitling. A common questionnaire for all tests has been established, and translated to all European languages where tests are administered. While benchmarking quality in all Pilot services is the final aim of tests, some tests will be designed to ascertain specific parameters as follows:

\section{A. Regarding subtitles:}

The impact of HbbTV subtitling implementations is multifold. In an HbbTV subtitling service it is feasible for the user to make selections of aspects of the subtitle presentation that previously were imposed and fixed by the broadcaster. For example the user might vary the font, the colours used, the location of the subtitles, the duration of subtitles (e.g. overriding out-cues) or the temporal presentation style (e.g. conversion of pop-on subtitles to a scrolling presentation at the Set-Top Box). This has important implications. What customisations may the user desire (or more importantly require)? What are the implications on authoring when the user can diverge from the assumed presentation?

It is proposed that 'user tests' are conducted to evaluate existing practises and conventions (or appropriate research is undertaken to identify supporting evidence as opposed to just accepting anecdotal wisdom). It is proposed that additional 'user tests' are conducted to establish the importance or utility of proposed new presentation options. These new presentation 'options' are possible in the HbbTV context as a result of the removal of many of the legacy limitations imposed upon subtitling by the traditional broadcast infrastructure. The following lists both existing practises and proposed new practises that might be subject to 'user tests' and their implementation in the testing scheme in the project: Subtitle delay, latency/duration, subtitle alignment: scene boundary, horizontal and vertical alignment, fonts, colour and reading subtitles on secondary screens.

On the other hand, when there is a lack of subtitles in the original media source, automatically generated subtitles are being integrated and tested within the pilots. For a limited domain of content, Large Vocabulary Continuous Speech Recognition (LVCSR) technology is used to generate intralingual subtitles for the original language of the media source. These subtitles will be then translated to a limited set of languages through a statistical Machine Translation system. The automatically generated intralingual and interlingual subtitles are being integrated in real-time in the HbbTV workflow, testing two different approaches: (1) A best-effort solution, where the subtitles will be delivery via Internet to be shown on top of the broadcasted content, with the delay introduced by the subtitle generation modules, and (2) an Internet-based solution, where the broadcasted video, audio and the generated subtitles will be encapsulated synchronously on an MPEG-DASH stream to be provided when the subtitles are generated.

\section{B. Regarding audio description:}

Audio description $(\mathrm{AD})$ is a spoken commentary. It therefore consists of more than just semantic information. To what extent might $\mathrm{AD}$ users infer meaning from the content of the description, and to what extent from the affective prosody (pace, tone, inflection) of the describer's voice? A Polish study explored the use of text-to-speech (TTS) as a cheaper alternative to Human Voice (HV) AD [2]. Although half the blind and partially sighted participants stated a preference for HV AD, the majority were willing to accept electronicallygenerated speech if it led to more source material being audio described. However, more recent studies with adults [3] and children [4] have shown that TTS AD can be less engaging and more difficult to understand. Fryer and Freeman [3] concluded that HV AD may be more important for blind audiences when accessing emotive content, such as drama, compared with purely informative genres, such as documentary. This study aims to test the applicability of TTS versus HV AD. 


\section{Regarding audio introductions:}

Audio introductions (AIs), also known as introductory notes, show notes or programme notes, have been used in opera and theatre since the early days of $\mathrm{AD}$. They are pieces of continuous prose, spoken by a single voice or a combination of voices lasting between 5 and 15 minutes. AIs aim to create a framework by which to understand the action; they have an information function providing relevant details such as running time, cast and production credits, as well as detailed descriptions of the locations, costumes and characters, and can convey a sense of visual style including camerawork and editing. Currently AIs are not provided for film or TV but a study by [5] suggested blind people would welcome the option to download them as an audio or text file from a website. HbbTV will offer a good opportunity to deliver AIs, as an alternative and complementary source of information which can be activated by the user at any time during the TV programme. This study aims at understanding user preferences and how they benefit from Audio Introductions in an HbbTV context.

\section{Regarding clean audio:}

Clean audio signal is generated by processing available audio signals to improve human voice intelligibility, either server-side or client-side, and provide that to the end-user. Tests will be aimed at understanding user preferences and how they benefit from the service and the possibility of having a dynamic end-user mix slider to be customised.

\section{E. Regarding audio subtitles:}

An audio subtitle service [6] may be automatically generated from subtitles and pre-processed scripts with textto-speech technology. Questions and tests will look to what extent might users infer meaning from the spoken content, quantifying how much information is missing, and to what extent an effective prosody enhances the reception.

\section{$F$. Regarding sign language:}

Because literature on the field is so scarce, the sign language interpreting tests in lab conditions will be preceded by quality data collection from sign language TV interpreters and signing Deaf TV consumers to understand the users preferences. These data will be used to help design the different conditions for the eye-tracking, memory and comprehension tests.

\section{CONCLUSIONS}

The Hbb4All project is well under way on testing access services at lab conditions with end users. New workflows and working conditions are also being tested, since HbbTV 2.0 for example opens the door to remote live sign language interpretation. Understanding automation of some services, such as subtitling, and their reception is also under way. At this early stage we can safely say Europeans respond evenly to access service reception on issues related to perception, such as subtitle reading speed, but slight differences are found when cultural issues are present. The richness of Europe regarding languages, language situations, cultures and traditions make difficult to unify reception results beyond perception, still a common result keeps on popping up in all tests: the need to raise awareness on media accessibility and the many access services already available.

\section{REFERENCES}

[1] United Nations - Department of Economic and Social Affairs, Population Division (2011). World Population Prospects: The 2010 Revision United Nations - Department of Economic and Social Affairs, Population Division (2011).

[2] Szarkowska, A. and Jankowska, A., 2011. Text-to-speech audio description: Towards wider availability of AD. Journal of Specialised Translation 15 (1) pp. $81-98$.

[3] Fryer, L., and Freeman, J., 2014. Can you feel what I'm saying? The impact of verbal information on emotion elicitation and presence in people with a visual impairment. Proceedings of the International Society on Presence Research. Vienna, March 2014.

[4] Zabrocka-Suwka, M., 2013. Audio description as a tool for creating the linguistic image of the world by blind children. Paper presented at the 5th Media for All Conference, Dubrovnik September 2013.

[5] Romero-Fresco, P. and Fryer, L.. 2013. Could Audio Described Films Benefit from Audio Introductions? An Audience Response Study. Journal of Visual Impairment and Blindness 107 (4) pp. 287-285.

[6] Braun, S. and Orero, P. 2010. Audio Description with Audio Subtitling - an emergent modality of audiovisual localisation. Perspectives: Studies in Translatology. 18 (3), pp.173-188. 